\title{
Asymptomatic Malaria And Glycemic Control Among Type 2 Diabetes Mellitus Patients In A Rural Tertiary Health Facility In Ido- Ekiti, Southwestern Nigeria- A Cross Sectional Study.
}

Azeez Oyemomi lbrahim ( $\square$ ibrahimazeez1434@gmail.com )

federal teaching hospital, ido-Ekiti, Ekiti State Nigeria

Tosin Anthony Agbesanwa

Ekiti State University College of Medicine

Segun Mathew Agboola

Afe Babalola University College of Medicine and Health Sciences

Olabode Muftau Shabi

Federal Teaching hospital, ido-Ekiti

Adewumi Oluwaserimi Ajetunmobi

Federal Teaching hospital, Ido-Ekiti

Waheed Olalekan Ismail

Obafemi Awolowo University Teaching Hospital, Ile-Ife

\section{Research}

Keywords: Asymptomatic malaria, glycemic control, T2DM, Ido- Ekiti, Southwestern Nigeria

Posted Date: January 5th, 2021

DOl: https://doi.org/10.21203/rs.3.rs-139006/v1

License: (c) (i) This work is licensed under a Creative Commons Attribution 4.0 International License.

Read Full License 


\section{Abstract}

Background: : Despite the high burden of Asymptomatic Malaria and Type 2 diabetes mellitus in subSaharan African, limited data exist regarding their co-occurrence and its associated clinical impacts of asymptomatic malaria parasitaemia on Type 2 diabetes mellitus in rural setting. The purpose of this study is to determine the prevalence of glycemic control and asymptomatic malaria parasitaemia; and to investigate the influence of socio-demographic characteristics and asymptomatic malaria parasitaemia on glycemic control among patients with Type 2 diabetes mellitus.

Methods: Hospital-based cross-sectional study was conducted on 150 T2DM patients at Federal Teaching Hospital, Ido-Ekiti, SouthWestern Nigeria, between April and September 2019. Systematic random sampling technique was adopted to recruit the respondents. Socio-demographic data were obtained using a semi- structured interviewer administered questionnaire and clinical files. Venous blood samples were collected and processed for glycosylated haemoglobin sugar estimation and malaria parasite detection by microscopy. Data were analyzed using SPSS version 20.0. Multivariate logistic regression analysis was used to identify the influence of asymptomatic malaria parasitaemia and the socio-demographic profile on glycemic control.

Results: The mean age, mean glycemic control and mean parasite density of the respondents were $64.8 \pm 11.1$ years, $6.9 \pm 2.1 \%$ and $1123.4 \pm 433.8$ respectively. The prevalence of poor glycemic control and asymptomatic malaria parasitaemia were $30.0 \%$ and $14.0 \%$ respectively. Old age, female gender, lack of formal education and asymptomatic malaria parasitaemia were associated with poor glycemic control.

Conclusion: The study revealed that Type 2 diabetes mellitus patients in Ido-Ekiti, Nigeria, harbor asymptomatic malaria parasitaemia and that Asymptomatic malaria parasitaemia, old age, female gender and illiteracy were negative predictors of glycemic control. Therefore, type 2 diabetes mellitus patient with poor glycemic control should be screened for asymptomatic malaria parasitaemia. Also, respondents with these identified predictors should be targeted with focus care and qualitative health education to improve their glycemic control level.

\section{Introduction:}

Malaria and T2DM pose an additional burden on the affected individuals and families, especially, the majority of patients in the rural settings where accessibility to quality health care is a challenge ${ }^{[1]}$ Despite the adoption of the global malaria elimination strategy through National Malaria Strategic Plan 20142020 in Nigeria, its desire effects are still far from being achieved. ${ }^{[2]}$ This is probably because high risk populations which often comprise adults with ASM, remain undiagnosed and serve as hotspots of malaria transmission with varying intensity. ${ }^{[3]} \mathrm{T} 2 \mathrm{DM}$ is a non- communicable disease that has attained great significance in the SSA with Nigeria being the most affected, malaria on the other hand seems to be concentrated in developing countries of the world. ${ }^{[3]}$ 
Ironically, report by the International Diabetes Federation (IDF) states that, the incidence of T2DM is increasing globally and by the year 2030 , about $80 \%$ of T2DM patients will be living in low predisposition. [4],[5] More worrisome than the absolute number of people with T2DM in Nigeria, is and middle income countries due to western lifestyles adaptation and genetic about $70-80 \%$ of the 4 million people who remain undiagnosed or untreated. ${ }^{[6]}$ This relatively large number poses a significant host and potential reservoirs of Plasmodium falciparum which has a negative impact on glycemic control. ${ }^{[7],[8]}$ The implications of the co-occurrence of T2DM with asymptomatic malaria in SSA will further deplete the poorly appropriated health budget of such region. The overall effect is that majority of patients present to health care facility with complications of advanced disease and its attendant high morbidity and mortality. ${ }^{[9]} \mathrm{A}$ study by Udoh et al in Nigeria showed that the prevalence of ASM among T2DM was 7.2\% and $16.8 \%$ using light microscopy and Polymerace Chain Reaction (PCR) respectively. ${ }^{[8]}$ The study further revealed that ASM was significantly associated with poor glycemic control $(p<0.04){ }^{[8]}$ The study also suggested that in SSA, the probability of co-occurrence of T2DM and ASM in the same individual is high, making the need to study the association between ASM and glycemic control among T2DM long overdue. ${ }^{[8]}$

To the best of our knowledge, there is currently paucity of data on the co-occurrence of ASM and T2DM among Nigeria patients, and where it exists, the study was done in the urban health care facility, and exclude the rural areas where malaria most often threatens the lives of people. ${ }^{[8]}$ Furthermore, the impact of such co-occurrence on diabetes management outcomes such as glycemic control remains unknown. In view of the predicted increased incidence of T2DM in SSA and the recent report of its co-occurrence with ASM. ${ }^{[7],[8]}$ The present study therefore set out to determine the level of glycaemic control, and the prevalence of ASM parasitaemia among T2DM patients; and to investigate the influence of sociodemographic characteristics and ASM parasitaemia on glycemic control among T2DM patients attending a rural tertiary health facility in Ido-Ekiti, Ekiti State, Southwestern Nigeria. Findings from this study would guide development of strategies to reduce the increasing T2DM trend and improve its case management. It would also contribute to the global elimination of ASM through case detection and treatment, in addition to integrated vector control efforts in transmission-prone areas. ${ }^{[10]}$

\section{Research questions}

This research answered the following questions;

1. What is the level of glycemic control among T2DM respondents attending FTH, Ido- Ekiti?,

2. What is the prevalence of ASM among the study respondents?

3. What is the relationship between sociodemographic characteristics of the respondents and their glycemic control?

4. What is the relationship between ASM parasitaemia and glycemic control among the study respondents?

Two null hypothesis were tested at 0.05 level of significance. 
1: There was no statistically significant relationship between socio-demographic characteristics and glycemic control of T2DM patients attending clinic in FTH, Ido-Ekiti?

2: There was no statistically significant relationship between presence of ASM parasitaemia and glycemic control of T2DM patients attending clinic in FTH, Ido-Ekiti?

\section{Materials And Methods:}

\section{Study area}

The study was conducted at the Family Medicine clinic of Federal Teaching Hospital (FTH), Ido-Ekiti, Southwestern Nigeria. The hospital (FTH) is located in the rural area of Ekiti State and about $30 \mathrm{~km}$ from Ado Ekiti, its state capital. ${ }^{[11]}$ The hospital offers secondary and tertiary care including specialist diabetic clinic to the people of its catchment area and its neigbouring states. The hospital was an accredited centre for postgraduate residency training in family medicine and other core clinical specialties. The people are mainly farmers and traders in the informal sector with a relatively small portion of the working population and retirees in the formal sector. In the study area, malaria transmission is perennial during the raining (April - October) and dry (November - March) seasons, with Plasmodium falciparum being the major causative agent. ${ }^{[11]}$ T2DM is also a health challenge among adult in Ekiti State and case of poor glycemic control among patients attending FTH had been reported. ${ }^{[12]}$

\section{Study population}

The study population comprised T2DM patients who have been receiving treatment at FTH, Ido-Ekiti.

\section{Inclusion criteria}

Consented T2DM patients on follow up for at least six months, who were asymptomatic for malaria, and were $\geq 95 \%$ treatment adherence in the previous three months (i.e, not missing more than six doses during the period for twice- daily or thrice-daily regimen).

\section{Exclusion criteria}

Respondents who were critically ill or have a major psychiatric illness and could not follow the study protocol. Other exclusion criteria were obese patient with Body Mass Index ( BMI) $\geq 30 \mathrm{Kg} / \mathrm{m} 2$ and hypertension (Mean Blood Pressure of $\geq 130 / 80 \mathrm{mmHg}$ for two measurements) and other diabetic related complications.

Sample size determination: Using the formula: $n=Z^{2} P q / d^{2} .{ }^{[13]}$ Where $n$ is the minimum sample size, $p$ is the 7.2\%,being the prevalence of ASM among T2DM patients on oral hypoglycemic agents for at least three months with a confidence Interval of $95 \%$ at $5 \%$ margin of error. ${ }^{[7]}$ Attrition of $10 \%$ was added to allow for unexpected data losses and drop-out, a total of 150 eligible respondents were enrolled. 


\section{Sampling technique}

A systematic random sampling technique was used to recruit the 150 respondents who fulfilled the inclusion criteria. The medical record of T2DM patients on follow up at Family Medicine clinic of FTH, Ido-Ekiti, showed that in the year 2018, an average of 10 T2DM patients were seen daily. This translated to 50 patients per week (Monday- Friday) and 800 (sample frame) patients over a period of four (16 weeks) months that the study was conducted. Using the formula, $K=N / n$, where $k$ is the sample interval, $\mathrm{N}$ the sample frame (800) and $\mathrm{n}$ represented the minimum sample size 150 . Therefore $\mathrm{k}$ was approximately 5 . The first respondent on each clinic day was chosen by simple random sampling and thereafter, every 5th respondent was chosen by systematic random sampling until the sample size of 150 was attained. A sticker was placed on each of the selected folders to avoid its re-sampling at the subsequent clinics.

\section{Recruitment procedure}

Respondents for the study were chosen using the inclusion criteria. Individual respondents were told about the methods and the objectives of the study and after which an informed consent was applied. This was either by appending signature or thumb printing. The data was collected using a pretested semistructured interviewer administered questionnaire which was developed by the researchers. The face and content validity of the questionnaire was established by two Consultant Family Physicians working at FTH, Ido-Ekiti. Construct validity and reliability coefficient $(\alpha=0.80)$ was established by using the Cronbach's alpha. The reliability coefficient 0.80 was within the acceptable range. The pretesting was conducted on 15 T2DM patients, also on follow up, and who were picked at random at the Family Medicine clinic of the Ekiti State University Teaching Hospital (EKSUTH), and lasted for two days. The pretesting was to show the fairness of the questionnaire and its applicability. Modifications of the questionnaire were done using the feedback from the pretest and validity assessement. With the assistance of the interpreter, translation of the questionnaire from English to local language was done for those who did not understand English Language while back translation to English language was later done to achieve response consistency. The questionnaire took about 10 minutes to complete.

\section{Data Collection Instruments}

The two instruments for data collection were the interviewer administered questionnaire and data collection form. The questionnaire sought information about the respondents' socio-demographic characteristics including the duration of their diabetes. The data was collected during the raining season over a period of 6 months (April- October 2019).

\section{Clinical parameters of the respondents:}

1. Blood pressure: With the aid of a reliable Accosson brand of Mercury Sphygmomanometer, blood pressure of the respondents was recorded twice after about 5 minutes apart and the Mean B.P of $\leq$ 130/80 mmHg was considered as controlled. The recording was done by a Senior Resident doctor. 
2. Body Mass Index: All respondents were directed to be in light clothing and barefooted for their weight and height measurement using an electronic weighing and a portable height board respectively. The weight was measured to the nearest $0.1 \mathrm{~kg}$ and height to the nearest $0.1 \mathrm{~cm}$. Normal BMI was defined as $18.5-24.9 \mathrm{~kg} / \mathrm{m} 2$, underweight as $<18.5 \mathrm{~kg} / \mathrm{m} 2$, overweight as $25-29.9 \mathrm{~kg} / \mathrm{m} 2$ and obese as $\geq 30.0 \mathrm{~kg} / \mathrm{m} 2$.

3. Glycosylated Hemoglobin ( HbAlc): After an overnight fast, $4 \mathrm{mls}$ of blood samples were collected through the venous route into fluoride and ethylenediaminetetra-acetic acid (EDTA) immunochemically on DCA 2000; HbAlc auto analyzer using kits supplied by Boehringer Mannheim auto analyser ${ }^{[14]}$ Accuracy of the test result was ensured using commercially prepared standards and control samples. Glycemic level was grouped into good control if HbAlc is $<7.0 \%$ (i.e $<$ $53 \mathrm{mmol} / \mathrm{mol}$ ) and uncontrolled if HbAlc is $>7.0 \%$ (i.e $>53 \mathrm{mmol} / \mathrm{mol}$ ). ${ }^{[15]}$

4. Microscopy: Two blood films, one thin and one thick was made from the blood sample collected for malaria parasitaemia. The thick and thin smears were prepared on clean, dry microscope glass slides and was allowed to dry. The thin smear was fixed in methanol and both smears were stained with $2 \%$ Giemsa BDH Laboratory supplies; Poole BH 15 ITD England. ${ }^{[16]}$ The slide was then viewed under a microscope using oil immersion at 100x magnification. Staining of the slide and parasite counting was done by a laboratory scientist of the hospital. The film was earlier viewed for the presence of parasite. A positive result means the presence of one parasite per 100 power field while a negative result means absence of parasite at 200 high power fields. The parasite density for the positive film was determined by counting the number of parasite in relation to the standard number of leucocyte per microlitre. If fewer than 10 parasites were counted, then, more of leucocytes were counted up to 500 and the result was recorded as the number of parasite per 500 leucocytes. Parasite density was then calculated using the formula; number of parasites over the number of leucocytes multiply by 8000 to give the number of parasites per microlitres of blood. A senior scientist was recruited to examine the slide for quality control, who also ensured that the authorized standard operating procedure for thin and thick blood films microscopy was strictly adhered to. ${ }^{[16]}$

\section{Definition of terms:}

1. T2DM Patient: Patient receiving documented treatment with anti-diabetes medication or having a glucose concentration of $\geq 7.0 \mathrm{mmol} /$ I. $^{[17]}$

2. Asymptomatic malaria (ASM): This was defined as being free of malaria symptoms ( including body temperature $\leq 37.2^{0} \mathrm{C}$ ) with presence of malaria parasitaemia as at the time of sample collection. ${ }^{[18]}$

\section{Data entry and analysis}

Data collected was checked, cleaned and entered into EPI Info Version 7.0 and exported to SPPSS version 22.0 for analysis. Computations, tabulations of proportions, percentage and other summary statistics were done. Chi-square analysis was used to determine the significance of association. Mean value was compared with students t-test. Chi- square (x2) and Fisher's exact test was used to compare the 
proportion of categorical variables. A p-value of $<0.05$ was considered significant. Binary logistic regression was done to identify the variables that were predictors of glycemic control and such variables were exported to the multivariate logistic regression model. The strength of the association between independent and dependent variables was measured using odds ratio and 95\% confidence Interval $(\mathrm{Cl})$ with significant level $(p$-value $<0.05)$.

\section{Ethical clearance, consideration and consent:}

Ethical approval for the study was received from the Ethics and Research Committee of FTH, Ido-Ekiti. The methods and objectives of the study were carefully explained to the participants individually, after which written informed consent was obtained either by appending signature or thumbprint for consent to participate in the study. For those participants who could not read or write, the questionnaire was translated from English language to their local language by an independent interpreter who served as their legal guardian while back translation to English language was later done to maintain response consistency. The respondents were free to refuse or disengage participation at any time without losing any benefit of care or favour to those that participated. Confidentiality and privacy were ensured throughout the study. The study was at no cost to the respondents

\section{Results:}

A total of one hundred and fifty respondents were studied. The mean age of the respondents was $64.8 \pm$ 11.1 (ranged 40-88) years. Most, 96 (64.0\%) were females and 109 (72.7\%) were married. Majority 121 (80.7\%) were from monogamous settings. Over one-third, 59 (39.3\%) were traders and $53(35.3 \%)$ were educated to the tertiary level. Majority, 140 (93.3\%) were Yorubas. More than half of the respondents 80 (53.3\%) were rural dwellers and 78 (52.0\%) lived below the poverty level. There was a statistically significant association between glycemic control and age $(p<0.001)$, gender $(p<0.001)$, marital status ( $p<$ $0.027)$, occupation $(p<0.005)$, level of education $(p<0.004)$ and income $(p<0.007),($ Table 1$)$. Over onethird, $60(40.0 \%)$ of the respondents had family history of diabetes and $92(61.3 \%)$ had been diabetic within the last ten years. There was a statistically significant association between glycemic control and duration of T2DM $(P<0.002)$, (Table 2$)$.

The mean glycemic control (HbAlc) was $6.9 \pm 2.1 \%$ and ranged from 4.4-16.0. A little above two third 105 (70.0\%) had good glycemic control, while $45(30.0 \%)$ had poor glycemic control. Therefore, the prevalence of poor glycemic control was $30 \%$ (Table 3.0).

Twenty one (14.0\%) of the respondents was positive for malaria parasitaemia. Therefore, the prevalence of ASM parasitaemia was $14 \%$. Furthermore, the mean parasite density of the respondents was 1123.4 \pm 433.8 and ranged from 480-1123.4 (Table 4.0). ). There was also a statistically significant association between glycemic control and ASM parasitaemia $(p<0.001)$ and parasite density $(p<0.001)$, (Table 5). 
Using multivariate binary logistic regression analysis, old age (OR, 6.334, 95\% Cl: 1.342-29.896), Female gender (OR,10.219, 95\% Cl : 2.069-50.473), illiteracy (OR,2.224, 95\% Cl: 1.052-19.643), presence of ASM (OR,92.563, 95\% Cl: 6.938-1233334.999) and parasite density of over 1,000 (OR,6.635, 95\% Cl: 1.69314.367) were the predictors of poor glycemic control (Table 6.0).

Table 1: Relationships between socio-demographic characteristics and glycemic control 


\begin{tabular}{|c|c|c|c|c|}
\hline \multirow{3}{*}{ Variable } & \multicolumn{4}{|c|}{ Glycemic Control } \\
\hline & Poor & Good & Chisquare(x2) & p-value \\
\hline & $n(\%)$ & $n(\%)$ & & \\
\hline Age group (in years) & & & 14.710 & $<0.001$ \\
\hline$<65$ & 8 (12.9) & $54(87.1)$ & & \\
\hline 65 and above & $37(42.0)$ & $51(58.0)$ & & \\
\hline Mean age $\pm S D$ & $70.0 \pm 8.4$ & $62.6 \pm 11.4$ & $3.958^{t}$ & $<0.001$ \\
\hline Gender & & & 11.662 & 0.001 \\
\hline Male & $7(13.0)$ & $47(87.0)$ & & \\
\hline Female & $38(39.6)$ & $58(60.4)$ & & \\
\hline Marital Status & & & 7.203 & 0.027 \\
\hline Married & $26(23.9)$ & $83(76.1)$ & & \\
\hline Separated & $2(50.0)$ & $2(50.0)$ & & \\
\hline Widowed & $17(45.9)$ & $20(54.1)$ & & \\
\hline Family type & & & 0.100 & 0.752 \\
\hline Monogamy & $37(30.6)$ & $84(69.4)$ & & \\
\hline Polygamous & $8(27.6)$ & $21(72.4)$ & & \\
\hline Occupation & & & 16.700 & 0.005 \\
\hline Civil Servant & $3(7.9)$ & $35(92.1)$ & & \\
\hline Artisan & $1(25.0)$ & $3(75.0)$ & & \\
\hline Farmer & $10(40.0)$ & $15(60.0)$ & & \\
\hline Trader & $26(44.1)$ & $33(55.9)$ & & \\
\hline Retiree & $3(18.8)$ & $13(81.2)$ & & \\
\hline Dependent & $2(25.0)$ & $6(75.0)$ & & \\
\hline Level of Education & & & 13.473 & 0.004 \\
\hline None & $15(44.1)$ & $19(55.9)$ & & \\
\hline Primary & $15(31.9)$ & $32(68.1)$ & & \\
\hline Secondary & $8(50.0)$ & $8(50.0)$ & & \\
\hline Tertiary & $7(13.2)$ & $46(86.8)$ & & \\
\hline
\end{tabular}




\begin{tabular}{|c|c|c|c|c|}
\hline \multicolumn{5}{|l|}{ Ethnicity } \\
\hline Yoruba & & & 1.071 & 0.784 \\
\hline Igbo & $42(30.0)$ & $98(70.0)$ & & \\
\hline Hausa & $3(37.5)$ & $5(62.5)$ & & \\
\hline Others & $0(0.0)$ & $1(100.0)$ & & \\
\hline Domicile & $0(0.0)$ & $1(100.0)$ & & \\
\hline Rural & & & 0.510 & 0.475 \\
\hline Urban & $26(32.5)$ & $54(67.5)$ & & \\
\hline Income & $19(27.1)$ & $51(72.9)$ & & \\
\hline$<$ N500/ day & & & 7.346 & 0.007 \\
\hline \multirow[t]{2}{*}{$\geq \mathrm{N} 500 /$ day } & 31 (39.7) & $47(60.3)$ & & \\
\hline & $14(19.4)$ & $58(80.6)$ & & \\
\hline
\end{tabular}

$t$ - Independent $t$ test

Table 2: Relationships between family history and duration of type $2 \mathrm{DM}$ and glycemic control

\begin{tabular}{|lllll|}
\hline Variable & \multicolumn{3}{l|}{ Glycemic Control } & \\
& Poor & Good & Chi square & p-value \\
& $\mathbf{n}(\%)$ & $\mathbf{n}(\%)$ & & \\
\hline Family History of Type 2 DM & & & 0.529 & 0.467 \\
\hline Yes & $16(26.7)$ & $44(73.3)$ & & \\
\hline No & $29(32.2)$ & $61(67.8)$ & & 0.002 \\
\hline Duration of Type 2 DM & & & 9.900 & \\
\hline$<10$ years & $19(20.7)$ & $73(79.3)$ & & \\
\hline$\geq 10$ years & $26(44.8)$ & $32(55.2)$ & & \\
\hline
\end{tabular}

Table 3: Prevalence of glycemic control among the respondents 


\begin{tabular}{|lll|}
\hline Variable & $\begin{array}{l}\text { Frequency } \\
\mathbf{N}=150\end{array}$ & $\begin{array}{l}\text { Percentage } \\
\text { (\%) }\end{array}$ \\
\hline Glycemic Control & & \\
\hline Poor & 45 & 30.0 \\
\hline Good & 105 & 70.0 \\
\hline Mean HbA1c $\pm S D(\%)$ & $6.9 \pm 2.1$ & \\
\hline HbA1c Range (\%) & $4.4-16.0$ \\
\hline
\end{tabular}

Table 4: Prevalence of asymptomatic malaria parasitaemia and parasite density among the respondents

\begin{tabular}{|lll|}
\hline Variable & $\begin{array}{l}\text { Frequency } \\
\mathbf{N}=150\end{array}$ & $\begin{array}{l}\text { Percentage } \\
\text { (\%) }\end{array}$ \\
\hline Asymptomatic Malaria Parasitaemia & & \\
\hline Present & 21 & 14.0 \\
\hline Absent & 129 & 86.0 \\
\hline Parasite Density & & \\
\hline Negative & 129 & 86.0 \\
\hline $101-1000$ & 9 & 6.0 \\
\hline $1001-2000$ & 12 & 8.0 \\
\hline Mean parasite density $\pm S D(n=21)$ & $1123.4 \pm 433.8$ & \\
\hline Parasite density range $(n=21)$ & $480-1123.4$ & \\
\hline
\end{tabular}

Table 5: Relationships between asymptomatic malaria parasitaemia, parasite density and glycemic control 


\begin{tabular}{|lllll|}
\hline Variable & \multicolumn{4}{l|}{ Glycemic Control } \\
& $\begin{array}{l}\text { Poor } \\
\text { n(\%) }\end{array}$ & Good & Chi square & p-value \\
\hline Asymptomatic Malaria Parasitaemia & & & 36.094 & $<0.001$ \\
\hline Present & $18(85.7)$ & $3(14.3)$ & & \\
\hline Absent & $27(20.9)$ & $102(79.1)$ & & \\
\hline Parasite Density & & & 36.566 & $<0.001$ \\
\hline Negative & $27(20.9)$ & $102(79.1)$ & & \\
\hline $101-1000$ & $7(77.8)$ & $2(22.2)$ & & \\
\hline $1001-2000$ & $11(91.7)$ & $1(8.3)$ & & \\
\hline
\end{tabular}

Table 6: Multivariate binary logistic regression analysis for predictors of poor glycemic control among respondents 


\begin{tabular}{|c|c|c|c|c|}
\hline \multirow[t]{2}{*}{ Variable } & \multirow[t]{2}{*}{ AOR } & \multicolumn{2}{|c|}{$95 \% \mathrm{Cl}$ for $\mathrm{AOR}$} & \multirow[t]{2}{*}{ p-value } \\
\hline & & LB & UB & \\
\hline \multicolumn{5}{|c|}{ Age group (in years) } \\
\hline$<65$ (ref) & 1.000 & & & \\
\hline 65 and above & 6.334 & 1.342 & 29.896 & 0.020 \\
\hline \multicolumn{5}{|l|}{ Gender } \\
\hline Male (ref) & 1.000 & & & \\
\hline Female & 10.219 & 2.069 & 50.473 & 0.004 \\
\hline \multicolumn{5}{|l|}{ Marital Status } \\
\hline Married (ref) & 1.000 & & & \\
\hline Separated & 7.931 & 0.476 & 132.263 & 0.149 \\
\hline Widowed & 0.563 & 0.158 & 2.010 & 0.376 \\
\hline \multicolumn{5}{|l|}{ Occupation } \\
\hline Civil Servant (ref) & 1.000 & & & \\
\hline Artisan & 33.227 & 0.837 & 1318.80 & 0.062 \\
\hline Farmer & 13.035 & 0.927 & 183.209 & 0.057 \\
\hline Trader & 5.068 & 0.517 & 49.668 & 0.163 \\
\hline Retiree & 0.193 & 0.014 & 2.630 & 0.217 \\
\hline Dependant & 3.919 & 0.189 & 81.213 & 3.919 \\
\hline \multicolumn{5}{|l|}{ Level of Education } \\
\hline None & 2.224 & 1.052 & 19.643 & 0.031 \\
\hline Primary & 1.201 & 0.146 & 3.687 & 0.347 \\
\hline Secondary & 0.953 & 0.025 & 1.986 & 0.893 \\
\hline Tertiary (ref) & 1.000 & & & \\
\hline \multicolumn{5}{|l|}{ Income } \\
\hline$<$ N500/ day & 1.467 & 0.359 & 6.002 & 0.594 \\
\hline$\geq \mathrm{N} 500 /$ day (ref) & 1.000 & & & \\
\hline \multicolumn{5}{|c|}{ Duration of Type 2 DM } \\
\hline$<10$ years (ref) & 1.000 & & & \\
\hline
\end{tabular}




\begin{tabular}{|lllll|}
$\geq$ & 2.270 & 0.725 & 7.107 & 0.159 \\
\hline Asymptomatic Malaria Parasitaemia & & & & \\
\hline Present & 92.563 & 6.938 & 1234.999 & 0.001 \\
\hline Absent (ref) & 1.000 & & & \\
Parasite Density & & & & \\
0 (ref) & 1.000 & & & \\
$101-1000$ & 2.249 & 0.766 & 5.369 & 0.268 \\
$1001-2000$ & 6.635 & 1.693 & 14.367 & 0.029 \\
\hline
\end{tabular}

ref - Reference Category

Adjusted Odd Ratio

$\mathrm{Cl}$-Confidence Interval $\quad$ UB - Upper Boundary

$A O R-$ $\angle B-$ Lower Boundary

\section{Discussion:}

The mean age of $64.8 \pm 11.1$ years found in this study was closed to $60.67 \pm 13.85$ obtained from a study by Gabriel et al, ${ }^{[19]}$ in the same study area and $60.96 \pm 10$ years recorded in another study in Abeokuta, Nigeria. ${ }^{[20]}$ This finding was not surprising because most of the chronic medical conditions develop during the middle age period.

In this study, the prevalence of respondents with poor glycemic control was $30.0 \%$. This was similar to finding by Ajayi et al in the same study area, where he reported a prevalence of $32.5 \%$ and $29.3 \%$ of the study population using American Diabetes Association (ADA) and IDF- Europe criteria respectively. ${ }^{[12]}$ However, our finding was less than report of a study by Ali et al conducted in the United States, where he found that the prevalence of poor glycemic control was $12.9 \% .{ }^{[21]}$ The difference with our finding could be due to difference in socio-economic characteristic of the respondents, diabetes management guideline and level of care. Furthermore, our finding was better than $47.0 \%$ reported by Danquah et a ${ }^{[7]}$ and $48.7 \%$ reported by Yigazu and Colleaque, ${ }^{[22]}$ based on HbAlc measurements. The difference between our study and the other studies could be due to variations in clinical characteristics of the respondents. For example, majority of the respondents in the other studies had obesity, dyslipidaemia and hypertension, whereas in our study, these co-founding factors have been excluded. ${ }^{[7],[22]}$ Previous studies have established the relationship between obesity, dyslipidaemia, hypertension and poor glycemic control. ${ }^{[7],[22]}$

In this study, 21 of the 150 afebrile T2DM respondents were found to harbor ASM parasitaemia. This finding is consistent with previous study conducted by Udoh et $\mathrm{al}_{1}{ }^{[8]}$ and Danquah et al, ${ }^{[7]}$ where they found that T2DM patients harbour Plasmodium falciparum asymptomatically. Therefore, these collective findings suggest that adult T2DM patients are potential reservoirs of malaria. Although, previous studies have not been able to define the actual reason for the presence of P. falciparum among T2DM patients, however, the risk increases with increasing glucose concentration and is a sign of biologic plausibility. ${ }^{[7]}$, 
${ }^{[8]}$ Probable reason for such risk could be due to impaired defence against the liver leading to a decrease in T-cell mediated immunity. ${ }^{[1]}$ Other suggested that P. falciparum grows faster in intensity with increased glucose availability as demonstrated invitro. ${ }^{[23]}$ The implication of this finding is that the increasing trend in the incidence of T2DM in developing countries of SSA may therefore further worsen the already increased number of person at risk of malaria infection. Thus, the burden of co-occurrence of T2DM and malaria in developing countries warrants further investigation into the significance of this finding. Our finding of $14.0 \%$ prevalence of ASM is greater than $7.2 \%$ reported in a study by Udoh et al, ${ }^{[8]}$ and $0.9 \%$ from a study by Danquah et al, ${ }^{[7]}$ using microscopy to detect ASM parasitaemia. The difference from our study could be due to difference in the sample size, study design and the geographical location between the study areas.

In the current study, the mean parasite density of the respondents was $1123.4 \pm 433.8 / \mathrm{ul}$. This finding is less than $1580 / \mathrm{ul}$ reported from a study by Udoh et $\mathrm{al}^{\left[{ }^{[8]}\right.}$ but higher than $880 / \mathrm{ul}$ reported from a study by Danquah et al. ${ }^{[7]}$ The difference with our finding could be due to the difference in the screening tools. This is because, in this study, we made use of microscopy to screen for ASM, whereas, studies by Udoh et al, ${ }^{[8]}$ and Danquah and colleaque, ${ }^{[7]}$ made use of Polymerase Chain Reaction (PCR). However, studies have established that PCR is more sensitive in identifying P. falciparum reservoirs than microscopy. ${ }^{[7],[8]}$ Therefore, using only microscopy to screen for ASM in this study, we may therefore have underestimated the burden of ASM among this set of patients.

In this study, using multivariate binary logistic regression analysis, ASM was found to be significantly associated with poor glycemic control. This finding is consistent with previous studies that have reported a statistically significant association between ASM parasitaemia and poor glycemic control among T2DM. ${ }^{[8],[24]}$ The implication of this finding to the global malaria elimination target by 2030 implies that in malaria endemic settings like this study area, if T2DM patients have poor glycemic control despite adherence to medication among other factors, screening for malaria parasitaemia is necessary to completely rule out $\mathrm{ASM} \cdot{ }^{[8],[25]}$

Report s from previous studies that investigated the influence of socio-demographic characteristic of the respondents on glycemic control have been inconsistent. [20],[26],[27] In this study, old age, female gender and lack of formal education were associated with poor glycemic control. This is consistent with previous studies, ${ }^{[26]}$ but inconsistent with other studies. ${ }^{[20],[27]}$ while other studies found no relationship. ${ }^{[28-30]}$ The reason for the poor glycemic control among the older patients in this study could be due to the fact that beta cell function begins to decline as age increases. This leads to decrease in insulin secretion and poor glucose utilization and therefore poor glycemic control. [27],[30]

Also, female gender was linked to poor glycemic control in this study as compared to other studies. This could be due to the differences in the study design, gender- based roles in the study area, and differences in the health -seeking behavior among the genders. ${ }^{[20],[30]}$ In view of this, interventional approaches should go beyond gender differences but patient - centre approach. ${ }^{[20]}$ 
In this study, lack of formal education was a predictor of poor glycemic control. Previous studies have linked informal education to lack of knowledge of the disease, leading to poor understanding of the treatment regimen and decrease in treatment outcomes. ${ }^{31,32}$ Therefore, continue health education and communication strategies by the stakeholders are needed as a measure to control the increasing trend of poor glycemic control among T2DM in the study area and Nigeria.

\section{Limitation:}

The study was limited by its cross sectional design and its sampling coverage. Therefore, finding from it cannot address the causal relationships between poor glycemic control and the factors found to be associated with it. Secondly, microscopy instead of PCR, was used to screen for malaria parasitaemia in this study. We may therefore have underestimated the burden of ASM among this set of patients.

\section{Strength of the study:}

It is noteworthy that some of the studies that have been conducted on glycemic control did not use HbAlc, which is an index of control. Hence, this study was conducted in Family Medicine clinic of a tertiary health facility in a rural area of Ekiti State using HbAlc as an index of control.

\section{Benefits of the study:}

1. The respondents with ASM parasitaemia were treated with safe antimalaria drug to prevent further complications associated with the co-occurrence of T2DM and malaria infection.

2. Regular follow up visit and detailed health education were given to the respondents with poor glycemic control in order to improve their glycemic control level.

\section{Implications for policy makers:}

The study revealed the co-existence of ASM and T2DM patients. Therefore, policy makers should canvass for more funding in the national health budget to cater for the integration of free ASM screening in the management of T2DM patients.

\section{Recommendations:}

1. Large community based studies are required to clarify fully the relationship between ASM parasitaemia and glycemic control among T2DM patients.

2. Further research is needed to identify other factors negating good glycemic control among the study population.

\section{Conclusion:}

This study found that in Ido- Ekiti, Southwestern Nigeria, T2DM patients are potential reservoirs of ASM parasitaemia and that ASM parasitaemia, old age, female gender and illiteracy were negative predictors of glycemic control. Therefore, respondents with these negative predictors should be targeted with focus 
care and qualitative health education to improve their glycemic control. The authors also recommend integration of ASM screening into the management of T2DM patients with poor glycemic control despite treatment adherence.

\section{Abbreviations}

ASM

Asymptomatic Malaria.

T2DM

Type 2 Diabetic Mellitus.

HbAlc

Glycosylated Haemoglobin

$\mathrm{FTH}$

Federal Teaching Hospital

\section{Declarations}

\section{Ethical clearance, consideration and consent:}

Ethical approval for the study was received from the Ethics and Research Committee of FTH, Ido-Ekiti. Informed consent was obtained from each respondents and participation was fully based on the willingness of the respondents. For those participants who could not read or write, the questionnaire was translated from English language to their local language by an independent interpreter who served as their legal guardian while back translation to English language was later done to maintain response consistency. The respondents were free to refuse or disengage participation at any time without losing any benefit of care or favour to those that participated. Confidentiality and privacy were ensured throughout the study. The study was at no cost to the respondents.

Consent for publication: Not Applicable

Availability of data and materials: The datasets for this study would be made available from the corresponding author on a reasonable request

Declaration of conflicts of interest: The authors declare that they have no conflicts of interest.

Funding: The researcher received no specific grant from any funding agency in the public, commercial or not-for- profit sectors.

\section{Authors' contribution:}

AOI- Conceptualization of the study, designed the study protocol, data acquisition and analysis and drafted the initial manuscript 
TAA- Literature review, data analysis and review of manuscript for intellectual content.

SMA- Critically revised the protocol for methodological and intellectual content.

OMS- Literature review, review of manuscript for intellectual content.

AOA- Literature review and review of manuscript for intellectual content

OWI- Literature review and review of manuscript for intellectual content.

All the authors read and approved the final version of the manuscript prior to submission.

\section{Acknowledgement:}

The authors expressed profound gratitude to the management of the Federal Teaching Hospital Ido- Ekiti for providing conducive atmosphere for the conduct of this research. Appreciation goes to the resident doctors and nurses at the Family Medicine Department.

Author's Information: Dr. Ibrahim AO is a medical Consultant and researcher at the Federal Teaching Hospital, Ido-Ekiti, Ekiti State, Southwestern Nigeria. His research interests include malaria prevention and control, emergency care and management, and lifestyle changes in the control of hypertension and diabetes in sub-Saharan Africa.

\section{References}

1. Muller LM, Gorter KJ, Hak E, Goudzwaard WL, Schellevis FG, Hoepelman Al, et al. Increased risk of common infections in patients with Type 1 and Type 2 diabetes mellitus. Clin Infect Dis. 2015;41:281-8. Doi:10.1086/431587.

2. Federal Ministry of Health. National Malaria Strategic Plan 2014-2020, Abuja. Nigeria: Federal Ministry of Health; 2014.

3. Sturrock HJ, Hsiang MS, Cohen JM, Smith DL, Green house B, Bousema T, et al. Targetting asymptomatic malaria infections: Active Surveillance in control and elimination. PLoS Med. 2013;10(6):e1001467. doi:10.1371.

4. Wild S, Roglic G, Green A, Sicree R, King H. Global prevalence of diabetes; estimates for the year 2000 and projections for the year 2030. Diabetes Care,2004:27: 1047-53 DOI: 10:2337/diacare. 27.5.1047.

5. Brancati FL, Kao WHL, Folsom AR, Watson RL, Szklo M. Incident type 2 diabetes mellitus in African American and white adults: the Atherosclerosis Risk in Communities study. JAMA. 2000;283:2253-9.

6. Olufemi A, Fasanmade. Samuel Dagogo- Jack. Diabetes care in Nigeria. Annals of Global Health. 2015;81(6):821-9.

7. Danquah I, Bedu-Addo G, Mockenhaupt FP. Type 2 Diabetes Mellitus and Increased Risk for Malaria Infection. Emerg Infect Dis. 2010;16(10):1601-4. 
8. Udoh BE, Iwalokun BA, Etukumana E, Amoo J. Asymptomatic falciparum malaria and its effect on Type 2 Diabetes Mellitus patients in Lagos, Nigeria. Saudi Journal of Medicine Medical Sciences. 2019;8:32-40.

9. Van Dieren S, Beulens JW, Vander Scchouw YT, Grobbee DE, Neal B. The global burden of diabetes and its complications. An emerging Pandemic. Eur J Cardiovas Prev Rehabil. 2010;17(1):3-8.

10. Bousema T, Okell L, Felger I, Drakeley C. Asymptomatic malaria infections. Nat Rev Microbiol. 2014;12:833-40.

11. Amase N, Dayo EL, Hellen EA, Aliu AA. Malaria parasitaemia among pregnant women possessing freely donated insecticide treated nets (ITNs) in Ado Ekiti, Nigeria. Global Journal of Biology Agriculture Health Sciences. 2014;3(1):86-90.

12. Ajayi EA, Ajayi AO, Olalekan OE. Treatment to targets in type 2 diabetes mellitus analysis of outpatients practice at a remote Western Nigeria hospital. IJMU. 2010;5(2):8-14.

13. Araoye MO. Subject Selection: Sample size determination In: Araoye MO. Research Methodology with Statistics for Health and Social Sciences. Nathadex Publishers; 2003: 117-118.

14. Hiar CE. Clinical Summary Report. Clinical Performance of DCA $2000+$ Hemoglobin Alc System SixMinute Assay. Bayer Diagnostic Division,1998: 1-7.

15. Buell C, Kermah D, Davidson MB. Utility of Alc for diabetes screening in the 1999-2004 NHANES population. Diabetes Care. 2007;30:2233-5.

16. Monif GRG. Infectious disease. In: Baker DA, editor. Obstetrics and gynaecology. 6th ed. New York: Partenon; 2004. pp. 280-6.

17. World Health Organization. Definition, diagnosis and Classification of diabetes mellitus and its complications: report of a WHO consultation. Part 1: diagnosis and classification of diabetes mellitus. Geneva: The Organization; 1999.

18. Oghagbon EK. Commentary. Improving persistently elevated HBAlc in diabetes mellitus patients in Nigeria. Ethn Dis. 2014;24:502-7.

19. Gabriel OE, Ajetumobi OA, Shabi OM, Adebara IO, Busari OA, Dada AS. Influence of family dynamics in medication adherence among hypertensive patients in a tertiary hospital in South-West Nigeria. JMSCR. 2017;5(7):25 146-25155.

20. Osuji NA, Ojo OS, Maloma SO, Ige Adegbola M, Egunjobi AO. Adeyemo AJ. Glycemic control and Practice of self care behaviors among people with T2DM in Nigeria. Plaid. 2019;5(1):1-18.

21. Ali MK, Mckeever BK, Imperatore G, Barker L, Gregg EW. Centers for Disease Control and Prevention (CDC). Characteristics associated with poor glycemic control among adults with self-reported diagnosed diabetes- National Health and Nutrition Examination Survey, United States, 2007-2010. MMWR Suppl. 2012;61(2):32-7.

22. Yigazu DM, Desse TA. Glycemic Control and associated factors among T2DM Patients at Shanan Gibe Hospital. Southwest Ethiopia. BMC Research Notes. 2017;10:597. 
23. Jensen MD, Conley M, Helstowski LD. Culture of Plasmodium falsiparum: the role of PH, g lucose, and lactate. J Parasitol. 1983;69:1060-7.

24. Ikekpeazu EJ, Neboh EE, Maduka IC, Nwagbara IJ, Nwobodo MW. Type 2 diabetes mellitus and malaria parasitaemia: effect of liver function tests. Asian Journal of Medical Science. 2010;2(5):214-7.

25. National Malaria Elimination Programme, National Population Commission, National Bureau of Statistics, and ICF International Nigeria Malaria Indicator Survey. 2015. Abuja and Rockville: National Malaria Elimination Programme, National Population Commission, and ICF International; 2016.

26. Wahba $\mathrm{H}$, Chang YF. Factors associated with glycemic control in patients with type 2 diabetes mellitus in rural areas of United States. Insulin. 2007;2:134-41.

27. Kautzky- Willer Al, Kosi L, Lin J, Mihaljevic R. Gender- based differences in glycemic control and hypoglycemia prevalence in patients with type 2 diabetes: Results from patient- level pooled data of six randomized controlled trials. Diabetes Obes Metab. 2015;17(6):533-40.

28. Shani M, Taylor TR, Vinker S, Lustman A, Erez R, Elhayany A, et al. Characteristics of diabetics with poor glycemic control who achieved good control. Am Board Fam Med. 2008;21:490-6.

29. Moreira ED, Neves RC, Nunes ZO, de Almeida MC, Mendes AB, Fittipaldi JA, et al. Glycemic control and its correlates in patients with diabetes in Venezuela: Result from a national survey. Diabetes Res Clin Pract. 2010;87:407-14.

30. Levey J, Atkinson AB, Bell PM, McCance DR, Hadden DR. Beta-cell deterioration determines the onset and rate of progression of secondary dietary failure in type 2 diabetes mellitus: The 10-year followup of the Belfast Diet Study. Diabet Med. 1998;15:290-6.

31. Saleem F, Hassali MA, Shafie AA, Atif M, Ul-hag N, Aljadhey H. Disease related knowledge and quality-of-life: a descriptive study focusing on hypertensive population in Pakistan. South Med Rev. 2012;5(1):47-52.

32. Youssef NF. Health-related quality of life, symptoms experience and perceived social support among patients with liver cirrhosis: A cross-sectional study in Egypt. School of Nursing, Midwifery and Health 2013: 14-269. 